\title{
SIZE DISTRIBUTION OF POSSIBLE DUST CARRIERS FOR THE EXTENDED RED EMISSION
}

\author{
D. P. Mahapatra, A. Chutjian, J. R. MachaceK, and R. S. Mangina \\ Jet Propulsion Laboratory, California Institute of Technology, Pasadena, CA 91109, USA; ara.chutjian@jpl.nasa.gov \\ Received 2014 February 3; accepted 2014 June 6; published 2014 July 16
}

\begin{abstract}
Power-law size distributions expected to be applicable to possible carriers of extended red emission (ERE) have been examined using Monte Carlo (MC) simulations. Si nanoparticles and some polycyclic aromatic hydrocarbon complexes such as oligoacene and oligorylenes with energy gaps close to $2 \mathrm{eV}$ have been considered. In the simplest case of unit quantum efficiency, the MC-generated size distributions are used to obtain photoluminescence (PL) spectra that are then corrected for dust extinction and reddening effects for comparison with observed ERE spectra. It is shown that a power-law size distribution with a decay exponent of $\alpha=7 / 2$, which closely agrees with starlight extinction data, fails to produce an ERE-like spectrum. However, size distributions with decay exponents of $\alpha=$ $19 / 12$ and $3 / 2$ are found to lead to acceptable spectra. Results indicate that energetic photon-induced breakup and competing aggregation effects dominate collisional effects in producing the observed steady-state mass distribution. It is shown that the peak wavelength of emission critically depends on the band gap, rather than cluster mass, which for oligoacenes and oligorylenes is widely different. The peak wavelength is also shown to be insensitive to dust attenuation.
\end{abstract}

Key word: dust, extinction

Online-only material: color figures

\section{INTRODUCTION}

The phenomenon of extended red emission (ERE) has been observed as a dust-mediated galaxy-prevalent process. ERE has been observed as a broad, structureless emission band extending from 500-900 nm, with a peak wavelength varying in the range of 600-850 nm. Summaries of ERE properties may be found in Witt et al. (1998), Ledoux et al. (2001), Whittet (2003), and Berné et al. (2008). The source of ERE has been ascribed to the presence of nanocrystalline silicon (Witt et al. 1998; Ledoux et al. 2001; Li \& Draine 2002; Smith \& Witt 2002) and/or to polycyclic aromatic hydrocarbons (PAHs; Bakes \& Tielens 1994; Vijh et al. 2004; Rhee et al. 2007; Berné et al. 2008).

It is now understood that ERE arises via a two-step process in which the first step involves the formation of a carrier species through the breakup of a precursor by ionization or dissociation with energetic, far-UV ( $>10 \mathrm{eV}$ ) photons (Witt et al. 2006). This is followed by near-UV to visible photon absorption by the carrier, resulting in ERE in the form of photoluminescence (PL) in the range of 500-900 nm (1.4-2.5 eV). The size distribution of the photoluminescent particles determines the peak wavelength and width of the ERE by quantum-confinement (QC) effects. QC enters through the size or mass reduction of the emitters, resulting in a widening of the gap between the highestoccupied molecular orbital (HOMO) and the lowest unoccupied molecular orbital (LUMO) - or the band gap, depending on whether the carriers are molecular species or solid nanoparticles.

One of the earlier models of ERE involved Si nanoparticles (SNP; Witt et al. 1998). Based on the laboratory-measured wavelength dependence of the PL using size-selected SNPs, a log-normal distribution with sizes in the range of 2.5-6.0 nm was proposed as the source of ERE (Ledoux et al. 2001). An objection to the SNP model is that energetic stellar photons induce breakup of these particles. This results in production of defect states in the form of dangling bonds that are known to quench the PL. There is an alternate model for the ERE based on PAH compounds that form a major constituent of interstellar dust. PAH di-cations $\left(\mathrm{PAH}^{++}\right)$with masses $<500 \mathrm{u}$ have been shown to have absorption and emission characteristics that make them strong candidates to be ERE carriers (Vijh et al. 2004; Witt et al. 2006; Malloci et al. 2007). The arguments have been primarily based on the PAH ionization potentials satisfying the two-stage breakup and excitation mechanism. However, nothing has been said about the size distribution of these PAH emitters and hence, unlike the case of SNPs, no ERE simulations have been carried out for them. Even for the case of SNPs, no correction for extinction by the interstellar dust has been considered. Extinction plays a significant role in shaping the ERE spectrum, and must be included in any simulation.

The size distribution of interstellar dust particles is assumed to be governed primarily by breakup and aggregation processes under a collisional environment (Mathis et al. 1977; Hayakawa \& Hayakawa 1988; O'Donnell \& Mathis 1997). Such cases, associated with non-equilibrium growth and aggregation, usually result in power-law distributions in the steady state with varied exponents (White 1982). The number of particles $(N)$ of size $(r)$ varies as $r^{-\alpha}$. In the large, conjugated PAH systems, total mass plays the role of size. In either case, the rate of particle growth in size or mass can be described by a master equation with three important terms: (1) a source term corresponding to continuous injection of the smallest fragment of SNPs or PAH complex generated through breakup of its larger size or mass; (2) a sink term that removes masses larger than a certain size; and (3) a term that involves the exchange of particles (mass), as given by a reaction kernel. In a collision scenario, this kernel describes the particle/mass exchange between sites and is usually a product of the relative thermal velocity and the collision cross section, both being dependent on the mass of colliding particles (Hayakawa \& Hayakawa 1988). When there is no sink leading to mass conservation, the master equation has bounded solutions where the size or mass distribution obeys a power law. Usually, this occurs over a mass range in which the decay exponent can be written as $\alpha=(3+\mu+v) / 2$ (Hayakawa \& Hayakawa 1988) or $3 / 2+\beta$ (White 1982). Violation of mass conservation leads to 


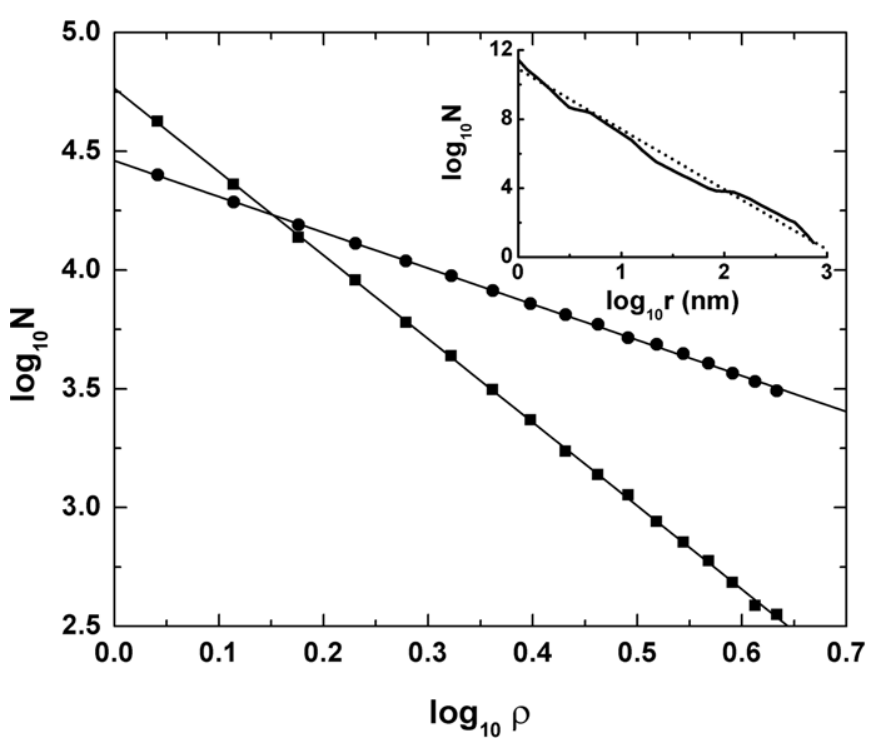

Figure 1. MC-generated scaled size distributions (in $\rho$ ) corresponding to powerlaw exponents $\alpha=7 / 2$ (filled squares) and $3 / 2$ (filled circles). The straight lines are fits to the data, leading to $\alpha$ values of 3.51 and 1.51 , respectively. The inset shows the results of Casuso \& Beckman (2010) as obtained for starlight extinction data. The dotted line in the inset corresponds to $\alpha=3.49 \pm 0.07$.

an exponential drop in size distribution (White 1982; Takayasu et al. 1988).

In an astrophysical scenario involving collision between thermalized grains, $\mu+v$ can have a value of $1 / 6$, leading to a value of $19 / 12$ for the exponent $\alpha$. This is very close to the value of $3 / 2$ that has been shown to emerge in cases where the rate of injection balances the rate of aggregation, with a constant reaction kernel independent of collision kinetics or mass (Takayasu 1989; Ben-Naim \& Krapivsky 2007). It appears to be a universal exponent valid for a large class of events, even applicable to biological species (Kuiri et al. 2008; Bonabeau \& Dagorn 1995; Bonabeau et al. 1999). It is therefore important to test whether there is a power-law-based size distribution for the ERE carriers, and if so, the value of the exponent. As argued herein, the size distributions are primarily governed by a dynamical interplay between fragmentation and growth, where collisional effects are less important. However, it is difficult to generate a situation in the laboratory where conditions may be suitable for producing normal or log-normal distributions with no competition between growth and breakup, unlike that expected in a harsh interstellar medium with particles moving and colliding in a high-flux region of energetic photons. In fact, as shown in Figure 1 (inset), extinction of electromagnetic radiation by galactic dust particles appears to follow a size distribution with $\alpha=7 / 2$ (Mathis et al. 1977; Casuso \& Beckman 2010). As shown by Vigil et al. (1988), such a size distribution can result in a situation where the reaction kernel has an explicit breakup term with a monomer loss rate proportional to the particle size. A power law with exponent $\alpha=7 / 2$ does appear, depending on initial conditions, when the relative strength of the fragmentation process over that of the coagulation process reaches a critical value. Based on the extinction data, an exponent of $7 / 2$ has been considered for SNPs proposed earlier to be responsible for ERE (Ledoux et al. 1998). It is shown herein that a broad spectrum, such as ERE with a peak around $700 \mathrm{~nm}$, cannot be generated from PL of small SNPs or PAH clusters with a size distribution given by $\alpha=7 / 2$. However, it is possible to get an ERE-like spectrum from the PL of the same carriers with a size distribution given by $\alpha=3 / 2$ or $19 / 12$ when $\beta$ or $\mu+v$ has a small value. Our analysis is based on the concept of QC that manifests itself with an energy gap (equal to the emission energy) that increases with size reduction. Emphasis is placed on the size distribution of possible carriers rather than on specifying a particular carrier.

\section{THE MODEL AND SIMULATION}

\subsection{Size Distribution and Emission Spectrum}

ERE spectra have been simulated herein using two generic cases of SNPs and PAH clusters. Both systems exhibit the confinement effect in energy depending on the particle size (nm) or cluster mass (u), respectively. Two sets of PAH clusters (oligoacenes and oligorylenes) are considered, with mass ranges of $128-328 \mathrm{u}$ and $252-748 \mathrm{u}$. The starting point in the calculation is the production of a particle distribution using the Monte Carlo (MC) method. In each case, $(1-2) \times 10^{6}$ particles are generated by inverting a $\rho^{-\alpha}$ distribution for exponents $\alpha=$ $7 / 2,19 / 12$, and $3 / 2$. This is done by generating a large number of uniformly distributed random numbers $\rho$ between 1 and 6 , with a probability of lying uniformly between 0 and 1 . All particles with weights or probabilities less than or equal to $\rho^{-\alpha}$ are selected for inclusion in the simulation. The number $\rho$ is then scaled appropriately to represent a diameter $(d)$ distribution between 1 and $6 \mathrm{~nm}$ for SNPs, or a mass $(m)$ distribution for masses lying between $128 \mathrm{u}$ and $748 \mathrm{u}$ for PAHs. Examples of the SNPs and PAH clusters are considered separately in the following.

The next step in the calculation is to generate the PL spectrum in terms of the emission energy $E_{\mathrm{em}}$ for the population of carriers under excitation by energetic photons. For SNPs, this is performed using the relation (Delerue et al. 1993; Ledoux et al. 2002),

$$
E_{\mathrm{em}}(e V)=E_{\mathrm{bg}}+3.73 / d^{1.39},
$$

where $E_{\mathrm{bg}}$ is the indirect band gap of $\mathrm{Si}$, equal to $1.17 \mathrm{eV}$. In the simplest scenario, one takes a single value for the excitation energy that is $3.4 \mathrm{eV}$ or higher, corresponding to the average direct band gap for crystalline $\mathrm{Si}$. This only places a restriction on the emission energy without entering into the calculation. Basically a particle will be excited to produce PL only when the excitation energy exceeds the energy of the band gap. By taking a higher value, one ensures that all the particles included in the ensemble are excited.

Unlike SNPs, PAH molecular systems have in general no such relation for the energy gap with size. In addition, a whole class of complex molecular systems comes under the PAH heading. The formation and growth of such systems in the interstellar medium has been discussed earlier (Rapacioli et al. 2006). However, based on the time-dependent (TD) density functional theory (DFT), there are electronic structure calculations in some oligoacenes $\left(\mathrm{C}_{4 n+2} \mathrm{H}_{2 n+4}\right.$; Malloci et al. 2007) and oligorylenes $\left(C_{10 n} H_{4 n+4}\right.$; Malloci et al. 2011), where $n$ is the number of carbon rings. Data are available for the HOMO-LUMO gaps for cluster masses in the range of 128-750 u in different series, which are adequate for our purposes. Most importantly, in charged or neutral PAH clusters, the DFT data on gaps are in good agreement with experimental data, where available. Following earlier suggestions (Witt et al. 2006), the first class of PAHs considered were the $\mathrm{PAH}^{++}$di-cations belonging to the acene series. Here, the energy gap, as given by the DFT for masses in the range of $128-328 \mathrm{u}$, fits to a form 


$$
E_{\mathrm{em}}(e V)=a / m^{b},
$$

where $a=60.35 \pm 1.33 \mathrm{eV}, b=0.618 \pm 0.004$, and $m$ is the cluster mass $(\mathrm{u})$. The calculation of the PL emission spectra for any power-law mass distribution with masses between $130 \mathrm{u}$ and $350 \mathrm{u}$ is then straightforward. For the $\mathrm{PAH}^{++}$clusters (as in the SNP case), a single incident energy is taken with a value equal to or greater than the maximum gap at the smallest cluster mass $(4.5 \mathrm{eV})$. The resulting emission spectra are then normalized to a constant peak height for comparison. The same procedure is followed with another class of PAH clusters, namely, the oligorylenes with masses in the range of 252-748 u, for which the band-gap energy varies between 3.0 and $1.5 \mathrm{eV}$, respectively. In the simplest scenario considered here, for both the SNP and PAH systems, the quantum efficiency for PL emission is taken to be unity. A lower value of the quantum efficiency, constant over the range of particles (as has been done in an earlier simulation; Ledoux et al. 2001), will not affect the results. It must further be mentioned that the PAH systems considered have discrete masses, whereas the MC-generated masses are not discrete. The effect of random mass can be considered to be the same as a fluctuation in energy around that expected for discrete masses. This can be considered to have its origin in structural defects in the species under consideration.

\subsection{Extinction and Reddening Effects}

For a realistic simulation of any ERE-like spectrum, one must take into account not only an appropriate particlesize distribution, but also the wavelength $(\lambda)$ dependence of the extinction of emitted photons along the line of sight. In the simplest picture, one can imagine the ERE photons encountering a foreground of dust absorbers with a wavelength-dependent optical depth $\tau_{\lambda}$. The observed transmitted intensity $I_{\lambda}$ can be written as $I_{\lambda}^{o} \exp \left(-\tau_{\lambda}\right)$, where $I_{\lambda}^{o}$ is the initial intensity. The optical depth $\tau_{\lambda}$ of the medium responsible for extinction is given by

$$
\tau_{\lambda}=0.921 A_{\lambda},
$$

where $A_{\lambda}$ is proportional to the product of the column density of the intervening dust and the scattering cross section (Whittet 2003). To connect with astronomically measured and parameterized quantities, Equation (3) can be written in terms of the color excess $E_{B-V}$ and the extinction parameter $R_{V}$ as

$$
\tau_{\lambda}=0.921\left(\frac{A_{\lambda}}{A_{V}}\right) R_{V} E_{B-V} .
$$

Universal extinction curves are available in the form of $A_{\lambda} / A_{V}$, from which $A_{\lambda}$ can be estimated using knowledge of $E_{B-V}$ and $R_{V}=A_{V} / E_{B-V}$ (Cardelli et al. 1989). However, in the wavelength range of 400-1000 $\mathrm{nm}$, the universal law leads to almost the same wavelength dependence for all values of $R_{V}$. Cardelli et al. (1989) have provided $R_{V}$ and $E_{B-V}$ data for a number of systems. The value $R_{V}=3.2$ is used, as it is valid for average Milky Way extinction. The color excess $E_{B-V}$ has been shown to vary in the range of $0.2-1.0$. A mid-range value of $E_{B-V}=0.5$ was adopted here, although several calculations were also carried out at values of 0.2 and 1.0.

\section{RESULTS AND DISCUSSIONS}

The MC-generated size distributions as derived for power-law exponents of $7 / 2$ and 3/2 are shown in Figure 1. Linear fits to the distribution data in double-log scales yield $\alpha=3.51 \pm 0.01$

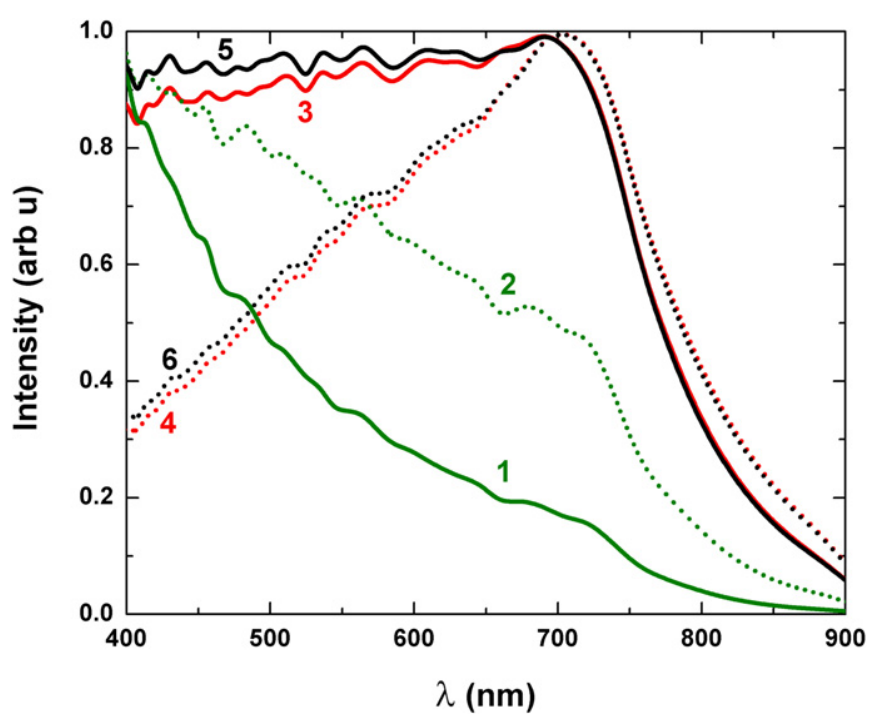

Figure 2. Simulated ERE spectra for $\mathrm{PAH}^{++}$with a high-mass cutoff at $310 \mathrm{u}$. The color excess $E_{B-V}$ and the extinction coefficient $R_{V}$ were taken as 0.5 and 3.3 , respectively. Continuous curves marked 1,3 , and 5 represent results for power-law exponents of $7 / 2,3 / 2$, and $19 / 12$, respectively, before extinction correction. Dotted curves marked 2, 4, and 6 show the corresponding results after extinction correction.

(A color version of this figure is available in the online journal.)

and $1.51 \pm 0.01$, in good agreement with the target values of 3.50 and 1.50. Also shown in Figure 1 (inset) is an interstellar dust grain size distribution derived from the results of Casuso \& Beckman (2010). A linear fit to these data corresponds to a distribution $N(r) \sim r^{-\alpha}$, where $\alpha=3.49 \pm 0.07$, appropriate for escape and shattering of large grains from the star-forming regions, and hence their loss from the particle distribution. This is close to the earlier results of Mathis et al. (1977), and was the motivation for using a power-law exponent $\alpha=7 / 2$ in our ERE simulation.

Using the procedure presented in the earlier sections, ERE spectra were simulated for $\mathrm{PAH}^{++}$systems using three exponents for the size distribution, $7 / 2,19 / 12$, and $3 / 2$, together with an exponential size cutoff at a maximum mass that could be varied depending on the requirement. The results obtained for the three exponents corresponding to a mass cutoff at $310 \mathrm{u}$ are shown in Figure 2. The raw simulated spectrum, for $\alpha=7 / 2$ prior to the extinction correction, is found to drop steadily from $400 \mathrm{~nm}$ to around $900 \mathrm{~nm}$, while that for $\alpha=3 / 2$ is seen to rise slowly from $400 \mathrm{~nm}$ to a peak at $700 \mathrm{~nm}$, finally dropping to zero at about $950 \mathrm{~nm}$. The ripplelike fluctuations in the spectra are due to random MC sampling and are not physical. Neither of the exponents gives rise to a PL spectrum with a peak in the ERE range of 600-800 nm. However, inclusion of the extinction effect results in a significant change in the spectral shape. The extinction-corrected spectra corresponding to all three distributions, normalized to the same peak value, are also shown in Figure 2. The final spectra corresponding to $\alpha=19 / 12$ and $3 / 2$ are seen to show a broad peak structure in the range of $600-900 \mathrm{~nm}$. No peak structure in the wavelength range of interest could be found for the distribution given by $\alpha=7 / 2$. This indicates that the mass distribution responsible for ERE carrier generation is not significantly affected by collisions among individual grains, or by grain relative velocities. Energetic photon-induced breakup would appear to produce smaller fragments that are injected into the ensemble at a constant rate. They are also 


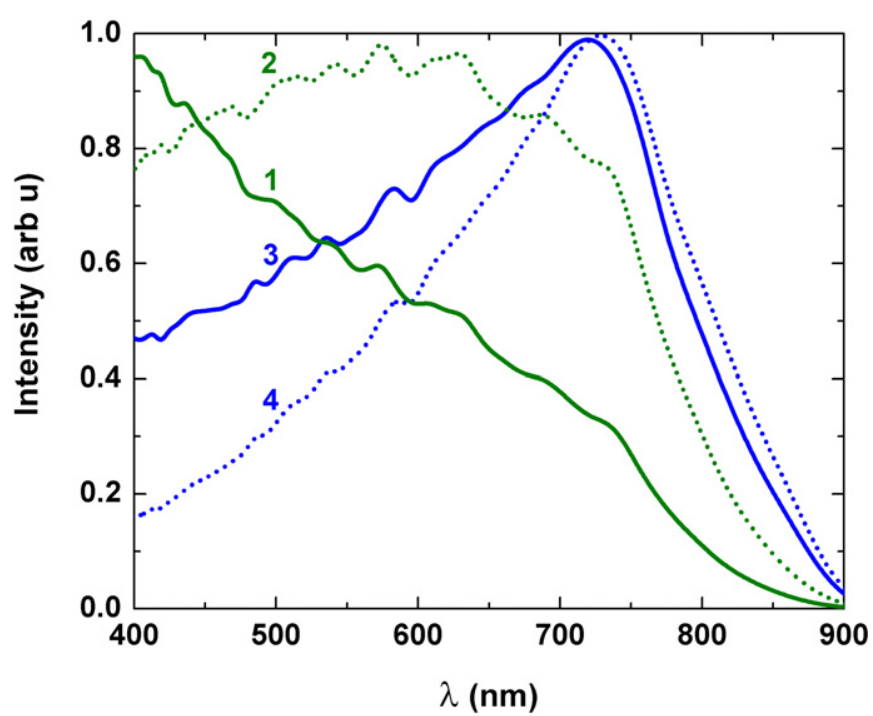

Figure 3. Simulated ERE spectra for SNPs with a large-diameter cutoff at $4.1 \mathrm{~nm}$. Continuous curves marked 1 and 3 correspond to power-law exponents of $7 / 2$ and $3 / 2$ before extinction correction, as indicated in Figure 2. Dotted curves marked 2 and 4 show the same data after extinction correction.

(A color version of this figure is available in the online journal.)

taken up at a similar rate by relatively smaller grains, leading to compensation of injection and aggregation rates with a nearly constant coagulation kernel. This is expected to produce a distribution that can be described by a power law with $\alpha=3 / 2$. A small component of collision and breakup in the kernel results in an exponent $\alpha=19 / 12$, differing only slightly from $\alpha=3 / 2$. Both exponents are found to lead to similar ERE-like PL spectra.

Shown in Figure 3 are results obtained on SNPs for size distributions obtained using $\alpha=7 / 2$ and $3 / 2$ with a size cutoff of $4.1 \mathrm{~nm}$. As with $\mathrm{PAH}^{++}$, use of $\alpha=19 / 12$ leads to results very similar to the exponent $3 / 2$. This is not shown in the figure to avoid crowding the spectra. Here again, the $7 / 2$ exponent is seen to fail regarding the production of an ERE-like spectrum, even after extinction correction. The value $\alpha=3 / 2$ gives an acceptable spectral shape. The same behavior was noted with massive neutral PAH clusters (oligorylenes) based on the HOMO-LUMO gap data given by the TD DFT (Malloci et al. 2011). The finding regarding the failure of $\alpha=7 / 2$ remains intact, whereas the value $\alpha=3 / 2$ leads to acceptable data. This is indicative of a general behavior for any carrier exhibiting QC with an energy gap around $2 \mathrm{eV}$, but there should be reasonable astrophysical justification in the choice of a carrier. Finally, shown in Figure 4 are simulated spectra obtained for the two PAH complexes oligoacene ${ }^{++}$and oligorylene $^{++}$, with mass cutoffs at $310 \mathrm{u}$ and $500 \mathrm{u}$, respectively. A power-law exponent of $3 / 2$ has been used in both cases. An observation-based spectrum obtained for the case of NGC 2327 is also included for comparison (Witt et al. 1998). The simulated data with a peak around $700 \mathrm{~nm}$ obtained for the PAH complexes oligoacene $^{++}$and oligorylene ${ }^{++}$resemble the observations and are similar to one another even though the mass ranges in the simulations are widely different.

The difference between the observations and simulations at shorter wavelengths is noteworthy. This discrepancy can be reduced using a larger extinction parameter $R_{V}$ and/or color excess $E_{B-V}$ (see Equation (4)). For completeness, the effect of decreasing and increasing $E_{B-V}$ from the adopted value of 0.5 was also examined. Shown in Figure 5 are the results of two

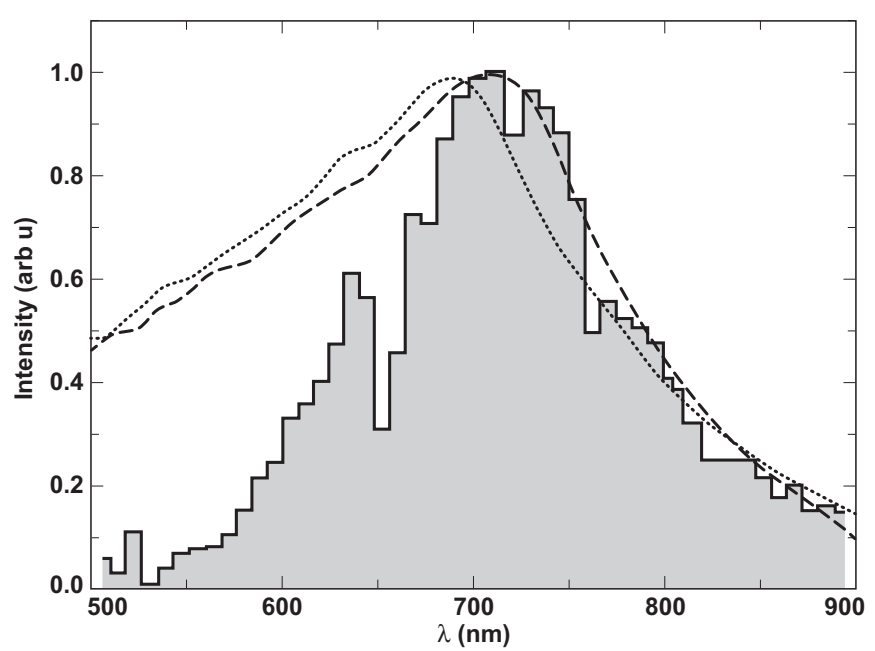

Figure 4. Simulated ERE spectra for di-cations of oligoacenes (dashed line) and oligorylenes (dotted line) based on the TD DFT calculations with mass cutoffs at $310 \mathrm{u}$ and $500 \mathrm{u}$, respectively, after extinction correction. Both spectra correspond to the same power-law exponent $\alpha=3 / 2$ with $E_{B-V}=0.5$. The filled histogram shows the observational spectrum on NGC 2327 (from Witt et al. 1998; see also Whittet 2003, Figure 6.14).

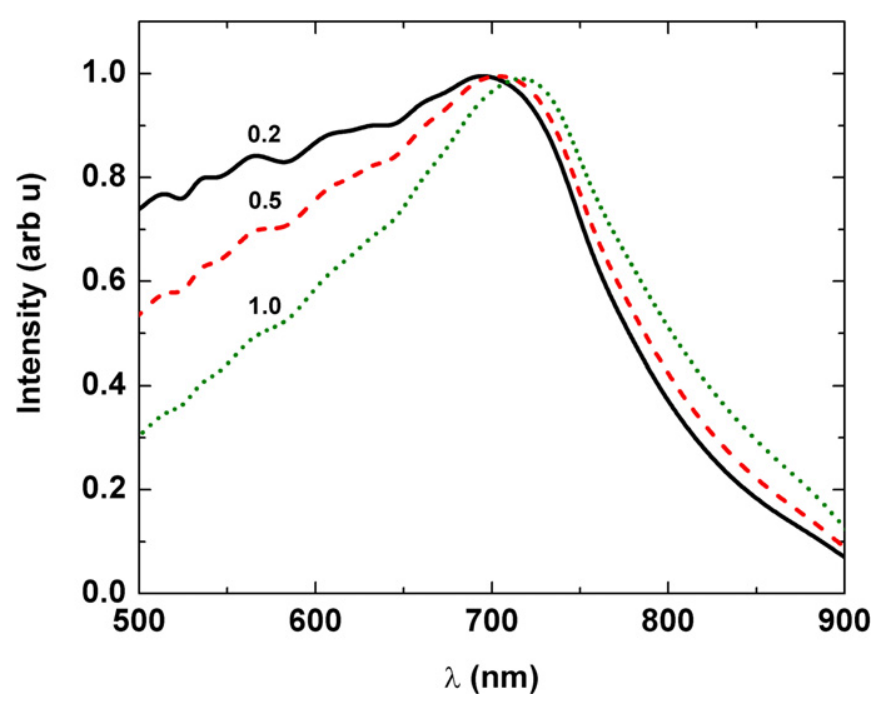

Figure 5. Simulated ERE spectra of oligoacene ${ }^{++}$with a mass cutoff at $310 \mathrm{u}$ for $\alpha=3 / 2$ and $E_{B-V}$ values of $0.2,0.5$, and 1.0, as indicated in the figure. (A color version of this figure is available in the online journal.)

additional calculations of ERE with decreased $\left(E_{B-V}=0.2\right)$ and increased $\left(E_{B-V}=1.0\right)$ dust attenuation in oligoacene ${ }^{++}$ for $\alpha=3 / 2$ and a mass cutoff at $310 \mathrm{u}$. The corresponding results for $E_{B-V}=0.5$ are also included for comparison. One can see the anticipated variation, with the highest value (1.0) leading to a fast fall in the lower wavelength region, resulting in better agreement with the observed ERE. This variation of $E_{B-V}$ from 0.2 to 1.0 is seen to have a negligible effect on the peak wavelength of the ERE. The observed difference is also related to the use of generalized size distributions that have been theoretically examined with respect to bounded solutions. Such a situation may not be valid for a physically realizable size distribution. One can see from the inset in Figure 1 that a more appropriate size distribution of particles resulting in starlight extinction (Casuso \& Beckman 2010) does not strictly agree with a 7/2 type distribution (Mathis et al. 1977), although they appear to be close to each other. 
It is important to point out that the HOMO-LUMO gap for oligorylene ${ }^{++}$at $500 \mathrm{u}$ is $1.83 \mathrm{eV}$, comparable to the gap of $1.9 \mathrm{eV}$ for oligocene ${ }^{++}$at $328 \mathrm{u}$, and both lead to ERE peaks near $700 \mathrm{~nm}$. This is also true for SNPs with a size cutoff around $4.1 \mathrm{~nm}$, where the band gap has a similar value. The above results indicate that a number of possible carriers with a size distribution given by a power-law exponent of $\alpha \sim 3 / 2$ can be used to simulate an ERE-like PL spectrum. The main criteria for the PL are that the quantum yield be sufficient for detection, that the HOMO-LUMO gap-or the band gap-be approximately $1.9 \mathrm{eV}$, and that there be an astrophysical rationale for the presence of the emitter in the astrophysical object (e.g.,, through the co-presence of a known vibrational emission band from the target). It is remarkable that there appears to be no mass restriction on the emitter. Different complexes can have different masses while still exhibiting the required band gap of $\sim 1.9 \mathrm{eV}$ for ERE production. As such, the suggestion that the source of ERE could be PAH di-cations or singly ionized PAH dimers is valid (Berné et al. 2008; Rhee et al. 2007), as is the suggestion that ERE spectra peaking at $~ 350 \mathrm{~nm}$ are indicators of species (SNPs, PAHs, or others) with band gaps around $3.5 \mathrm{eV}$ (Vijh et al. 2004; Nayfeh et al. 2005; Wada et al. 2009).

To conclude, using power-law distributions, simulations have been carried out to unravel the aggregation dynamics of ERE carriers. SNPs and representative PAH particles were used as astronomically relevant targets. The simulations were carried out using general principles without requiring details of the particle-particle interactions. Even then it has been possible to rule out an $\alpha=7 / 2$ power-law distribution shown to be valid for larger particles. Results indicate the validity of a steadystate scenario as given by the generalized exponent $\alpha=3 / 2$. The band gap is the dominant parameter deciding the peak position. For all emitters, irrespective of mass, a band gap of about $2 \mathrm{eV}$ can result in a peak structure around $700 \mathrm{~nm}$ in the PL as observed for ERE. The value $\alpha=3 / 2$ for the powerlaw exponent is a result of photon-induced breakup leading to an injection of small units of mass that are picked up by larger fragments in a mass-independent aggregation that leads to a dynamically stable particle distribution. The analysis also suggests a negligible mass-dependent collisional effect between particles that lead to ERE.

We thank M.P. Das for helpful discussions. D.P.M. acknowledges support from NASA through the NASA Senior Research Associate Program managed by the Oak Ridge Associated Universities, USA. This research was carried out at JPL/Caltech under contract with the National Aeronautics and Space Administration.

\section{REFERENCES}

Bakes, E. L. O., \& Tielens, A. G. G. M. 1994, ApJ, 427, 822 Ben-Naim, E., \& Krapivsky, P. L. 2007, PhRvB, 75, 011103

Berné, O., Joblin, C., Rapacioli, M., et al. 2008, A\&A, 479, L41

Bonabeau, E., \& Dagorn, L. 1995, PhRvE, 51, R5200

Bonabeau, E., Dagorn, L., \& Fréon, P. 1999, PNAS, 96, 4472

Cardelli, J. A., Clayton, G. C., \& Mathis, J. S. 1989, ApJ, 345, 245

Casuso, E., \& Beckman, J. E. 2010, AJ, 139, 1406

Delerue, C., Allan, G., \& Lannoo, M. 1993, PhRvB, 48, 11024

Hayakawa, H., \& Hayakawa, S. 1988, PASJ, 40, 341

Kuiri, P. K., Joseph, B., Lenka, H. P., et al. 2008, PhRvL, 100, 245501

Ledoux, G., Ehbrecht, M., Guillois, O., et al. 1998, A\&A, 333, L39

Ledoux, G., Gong, J., Huisken, F., Guillois, O., \& Raynaud, C. 2002, ApPhL, 80,4834

Ledoux, G., Guillois, O., Huisken, F., et al. 2001, A\&A, 377, 707

Li, A., \& Draine, B. T. 2002, ApJ, 564, 803

Malloci, G., Cappellini, G., Mulas, G., \& Mattoni, A. 2011, CP, 384, 19

Malloci, G., Mulas, G., Cappellini, G., \& Joblin, C. 2007, CP, 340, 43

Mathis, J. S., Rumpl, W., \& Nordsieck, K. H. 1977, ApJ, 217, 425

Nayfeh, M. H., Habbal, S. R., \& Rao, S. 2005, ApJL, 621, L121

O’Donnell, J. E., \& Mathis, J. S. 1997, ApJ, 479, 806

Rapacioli, M., Calvo, F., Joblin, C., et al. 2006, A\&A, 460, 519

Rhee, Y. M., Lee, T. J., Gudipati, M. S., Allamandola, L. J., \& Head-Gordon, M. 2007, PNAS, 104, 5274

Smith, T. L., \& Witt, A. N. 2002, ApJ, 565, 304

Takayasu, H. 1989, PhRvL, 63, 2563

Takayasu, H., Nishikawa, I., \& Tasaki, H. 1988, PhRvA, 37, 3110

Vigil, R. D., Ziff, R. M., \& Lu, B. 1988, PhRvB, 38, 942

Vijh, U. P., Witt, A. N., \& Gordon, K. D. 2004, ApJL, 606, L65

Wada, S., Mizutani, Y., Narisawa, T., \& Tokunaga, A. T. 2009, ApJ, 690, 111

White, W. H. 1982, JCIS, 87, 204

Whittet, D. C. B. 2003, Dust in the Galactic Environment (Bristol, UK: IOP Publishing), 222

Witt, A. N., Gordon, K. D., \& Furton, D. G. 1998, ApJL, 501, L111

Witt, A. N., Gordon, K. D., Vijh, U. P., et al. 2006, ApJ, 636, 303 